\title{
Multilayered Polymer Capsules with Switchable Permeability
}

\author{
Kristian Kempe, Ka Fung Noi, Sher Leen Ng, Markus Müllner, and Frank Caruso* \\ Department of Chemical and Biomolecular Engineering, The University of Melbourne, Victoria \\ 3010, Australia
}

\begin{abstract}
The preparation of layer-by-layer (LbL) polymer capsules stabilized by a combination of coppercatalyzed azide-alkyne cycloaddition (CuAAC, "click chemistry") and disulfide cross-linking is reported. The capsules obtained possess gated permeability due to the reversible nature of the disulfide cross-linking. Poly(methacrylic acid) (PMA) was modified with different ratios of alkyne and protected thiol functionalities $\left(\mathrm{PMA}_{\mathrm{Alk}, \mathrm{SH}}\right)$. Exploiting hydrogen bonding interactions, multilayered films were prepared by the alternate deposition of PMA $\mathrm{Alk}, \mathrm{SH}_{\text {(hydrogen bonding donor) }}$ and poly( $N$-vinylpyrrolidone) (PVPON; hydrogen bonding acceptor), yielding stable PMA $\mathrm{Alk}_{\mathrm{SH}}$ capsules. Capsule pH responsive behavior and permeability, using a series of different size labeled dextrans, were investigated in both the “closed” state (disulfide cross-linking intact) and “open” state (cleaved disulfide bonds). To demonstrate the potential of these gateable systems, post-loading studies with fluorescent 45-base pair (bp) linear double-stranded (ds) DNA were performed. Fluorescence studies revealed the potential of these capsules to reversibly encapsulate cargo: cleavage and reformation of the disulfide groups resulted in reversible permeability to the DNA.
\end{abstract}

\section{KEYWORDS}

layer-by-layer, capsules, disulfide chemistry, click chemistry, permeability.

\section{INTRODUCTION}

The design of "smart" nanostructured materials that respond to changes in their environment is of high interest for applications ranging from drug delivery to microreactors. ${ }^{1-3}$ The layer-by-layer 
(LbL) technique is a robust method for the fabrication of multilayer thin films and the nanoscale engineered assembly of materials. In particular, the versatility of this process is demonstrated by its application to a variety of templates, such as planar surfaces, ${ }^{4,5}$ particles, ${ }^{6}$ red blood cells, ${ }^{7}$ and emulsions. ${ }^{8}$ To date, different LbL assembly strategies have been applied, ${ }^{9}$ including the deposition of polymer layers through electrostatic, ${ }^{10}$ hydrogen bonding, ${ }^{11}$ and covalent interactions. ${ }^{12}$ A distinct advantage of LbL assembly is the precise control over film properties, such as thickness and morphology. In the past years, the development of LbL capsules that respond to specific stimuli for therapeutic delivery applications has gained significant interest. ${ }^{13}$ Pre- and post-loading approaches allow the encapsulation of a range therapeutic or sensing cargo. 2, 14, 15 However, systems which exhibit switchable layer/wall permeability are of particular interest for both loading/release and microreactor applications. The permeability of LbL capsules can be controlled by a number of factors, such as choice of polymer, number of polymer layers, and surface modification. Depending on the material, the capsules can be assembled to be responsive to external stimuli such as redox, ${ }^{16}$ salt, $^{17}$ temperature, $^{18,}{ }^{19}$ and $\mathrm{pH}^{20-22}$ For example, Tong et al. synthesized poly(acrylic acid)/branched poly(ethyleneimine) capsules that were cross-linked using glutaraldehyde. ${ }^{23}$ These $^{2}$ capsules displayed pH-responsive permeability; below pH 6, $2000 \mathrm{~g} \mathrm{~mol}^{-1}$ dextran could readily diffuse into the interior of the capsules, while above pH 6 it was excluded. Despite such studies, to the best of our knowledge, there are no reports on the design and assembly of LbL capsules that exhibit gated permeability based on reversible covalent linkages.

In the present study, a biologically relevant mechanism for reversible capsule permeability, based on a combination of non-reducible copper-catalyzed azide-alkyne cycloaddition (CuAAC) and reversible disulfide bond formation cross-linking, is presented. Disulfides are of interest as a triggered release system, as they are stable in vivo but can be degraded to their thiol components intracellularly. ${ }^{24}$ Recently, disulfide cross-linking has been used to stabilize poly(methacrylic acid) (PMA) capsules, which were formed by the alternate assembly of a thiol-modified PMA (PMA $\left.{ }_{\mathrm{SH}}\right)$ with poly( $N$-vinylpyrrolidone) (PVPON), followed by oxidation of the thiol groups in the film and 
removal of PVPON at physiological $\mathrm{pH}^{25}$ Such disulfide cross-linked PMA capsules have been used to release peptide cargo both in vitro ${ }^{26}$ and in vivo ${ }^{27}$ upon disulfide cleavage. However, as the disulfide bonds provide the sole source of stabilization for the multilayers, the capsules disassemble upon disulfide cleavage. ${ }^{28}$ To control the permeability of the capsule systems while retaining their integrity, another stabilization factor needs to be incorporated into the multilayers. Recently, reactions summarized under the term "click chemistry" have generated interest for the synthesis of tailor-made materials due to the highly selective, mild and quantitative nature of these reactions. ${ }^{29-31}$ To date, the "click" approach has been exploited for the synthesis of various architectures. ${ }^{32}$ In particular, the classical "click reaction" (CuAAC), ${ }^{33}$ has been used to assemble a range of LbL films, and to functionalize the resulting films to tune their surface properties. ${ }^{34}$ In contrast to films constructed using disulfide chemistry, films with CuAAC linkages are stable under different redox environments. Thus, using reversible disulfide cross-linkers in combination with other nondegradable cross-linkers (e.g., via click chemistry) would enable the design of reversibly permeable, gated capsule systems. Such capsule systems are expected to be switchable between a "closed" state (disulfide bonds) and an "open" state (free thiol groups).

Herein, the synthesis of bifunctional PMA and its application for the assembly of LbL capsules with both disulfide and CuAAC cross-links is reported. The combination of these two chemistries offers new possibilities for responsive, nanoengineered capsules. To this end, the preparation and characterization of respective polymers are described in detail. Polymers with different degrees of functionalization were synthesized and examined for their potential to form stable capsules. Permeability studies with dextrans of different molar mass provide insights into the switchable character of the systems. Furthermore, the ability of these capsule systems to load and release DNA is demonstrated. This control of permeability, through disulfide exchange chemistry, could be useful for the application of LbL capsules in drug delivery, as it allows post-loading of sensitive cargo and offers the potential to release the cargo specifically within the reducing intracellular environment of cells. 


\section{EXPERIMENTAL SECTION}

\section{Materials and Instrumentation}

Silica particles of $1.16 \mu \mathrm{m}$ diameter were purchased from MicroParticles GmbH (Germany) as a 5 wt\% suspension and were used as received. Poly(methacrylic acid, sodium salt) (PMA, 30 wt.\%), $\mathrm{M}_{\mathrm{w}} 15 \mathrm{kDa}$, was purchased from Polysciences (USA). Poly( $N$-vinylpyrrolidone) (PVPON), $\mathrm{M}_{\mathrm{w}} 10$ $\mathrm{kDa}$, fluorescein isothiocyanate-dextran, $\mathrm{M}_{\mathrm{W}}$ 10, 59-77, 250, 500, and $2000 \mathrm{kDa}$, dithiothreitol (DTT), 4-(4,6-dimethoxy-1,3,5-triazin-2-yl)-4-methylmorpholinium chloride (DMTMM), propargylamine hydrochloride, $N$-chloro- $p$-toluenesulfonamide sodium salt hydrate (chloramine-T hydrate), 2,2'-dithiodipyridine (DTDP), phosphate buffered saline (PBS) tablets, and buffering salts were purchased from Sigma-Aldrich and used as received. 2-(pyridylthio)-ethylamine hydrochloride was obtained from Speed Chemical (China), and azido-dPEG $\mathrm{A}_{3}$-amine was obtained from Quanta BioDesign (USA). Alexa Fluor 647 azide, and triethylammonium salt (AF647 Az) were obtained from Invitrogen. DNA sequences were obtained from GeneWorks (Adelaide, Australia). High-purity water with a resistivity greater than $18 \mathrm{M} \Omega \mathrm{cm}$ was obtained from an in-line Millipore RiOs/Origin water purification system (Milli-Q water). A stock solution of saline sodium citrate (SSC buffer) was prepared as outlined previously. ${ }^{35}$

Fluorescence microscopy images of the capsules were performed with an inverted Olympus IX71 microscope equipped with a DIC slider (U-DICT, Olympus) and a 60× objective lens (Olympus UPLFL20/0.5 N.A., W.D. 1.6) was used to image capsules. A CCD camera (Cool SNAP $f x$, Photometrics, Tucson, AZ) was mounted on the left hand port of the microscope. Fluorescence images were illuminated with an Hg arc lamp using a UF1032 filter cube. Confocal laser scanning microscopy (CLSM) images were taken using a Leica TCS-SP2 confocal laser scanning microscope using a Picoquant 405-nm pulsed diode laser as the excitation source. Fluorescent click NPS were imaged in x-y mode with a 63x planapochromatic oil immersion objective using a PMT gain of 550 $\mathrm{V}$, a digital zoom of $2 \times$, a line frequency of $400 \mathrm{~Hz}$, and $4 \mathrm{x}$ line averaging in 12-bit acquisition mode. Images were analyzed using Image J $(1.47 \mathrm{v})$. AFM measurements were carried out with a JPK NanoWizard II BioAFM (JPK Instruments AG, Germany). Glass slides (Livingstone, Australia) 
were cleaned with piranha solution (Caution! Piranha solution reacts violently with organic compounds. Extreme care should be taken when handling piranha solution, and only small quantities should be prepared.) The sample solution ( $4 \mu \mathrm{L})$ was placed onto the glass slide and airdried. The images were obtained in intermittent contact mode with a scanning rate of $0.5 \mathrm{~Hz}$. Tapping mode cantilevers (BudgetSensors, Tap300Al-G, resonance frequency; 300 kHz, force constant; $\sim 40 \mathrm{~N} \mathrm{~m}^{-1}$ ) with a digital resolution of $512 \times 512$ pixels were used.

\section{Polymer Post-modifications}

$\mathrm{PMA}_{\mathrm{Alk}, \mathrm{SH}}$ was synthesized by either first introducing the alkyne or the protected thiol (PDA) functionality first. The functionalization with propargyl amine hydrochloride was performed in Milli-Q water, whereas PBS was used as a medium for PDA functionalization. To an aqueous PMA solution (30 wt\%), DMTMM (20 $\mathrm{g} \mathrm{L}^{-1}, 2$-fold excess) was added and stirred for 15 min at room temperature. Subsequently, propargyl amine hydrochloride or PDA (20 g L $\mathrm{L}^{-1}$, 1.5-fold excess) was added and stirred over night at room temperature to obtain PMA Alk and PMAPDA, respectively. The polymer was purified by extensive dialysis against Milli-Q water (3-4 days, with at least two water exchanges per day) and was recovered by freeze-drying. ${ }^{1} \mathrm{H}$ NMR: $\delta_{\mathrm{H}}\left(400 \mathrm{MHz} ; \mathrm{D}_{2} \mathrm{O}\right)$ : $\mathrm{PMA}_{\mathrm{Alk}}$ : 0.5-1.25 $\left(\mathrm{CH}_{3}\right)$, 1.25-2.2 ( $\mathrm{CH}_{2}$ backbone), 2.3-2.54 ( $\mathrm{CH}$ alkyne), 3.6-3.85 ( $\mathrm{NH}^{\left.-\mathrm{CH}_{2}\right) \text {. PMA }} \mathrm{PDA}_{0}$ 0.5$1.25\left(\mathrm{CH}_{3}\right), 1.25-2.2$ ( $\mathrm{CH}_{2}$ backbone), 2.65-3 (S-CH$)$, 3.2-3.5 $\left(\mathrm{NH}-\mathrm{CH}_{2}\right)$, 7.0-7.3, 7.5-7.9, 8.25-8.4 (CH pyridine).

The second functionalization was introduced accordingly. The mono-functional PMA was dissolved in Milli-Q water (20 mg mL ${ }^{-1}$ ) and DMTMM (20 mg mL ${ }^{-1}, 2$-fold excess) and the second reagent (20 mg mL $\mathrm{m}^{-1}$, 1.5-fold excess) were added and the solution was kept overnight at room temperature. The resulting polymer was purified by passing it through a NAP-25 column, and it was recovered by freeze-drying. ${ }^{1} \mathrm{H} \quad \mathrm{NMR}: \delta_{\mathrm{H}}\left(400 \mathrm{MHz} ; \mathrm{D}_{2} \mathrm{O}\right): \mathrm{PMA}_{\mathrm{Alk}, \mathrm{PDA}}: 0.5-1.25\left(\mathrm{CH}_{3}\right), 1.25-2.2\left(\mathrm{CH}_{2}\right.$ backbone), 2.34-2.54 ( $\mathrm{CH}$ alkyne), 2.65-3 (S-CH$), 3.2-3.5\left(\mathrm{NH}-\mathrm{CH}_{2}\right), 3.6-3.85\left(\mathrm{NH}-\mathrm{CH}_{2}\right.$ alkyne), 7.0-7.3, 7.5-7.9, 8.25-8.4 (CH pyridine). Degree of functionalization (DF) was determined by ${ }^{1} \mathrm{H}$ 
NMR spectroscopy. A detailed overview of the amounts used and the DF values are provided in Table 1.

\section{LbL Assembly on Silica Particles}

Prior to use, PMA Alk,PDA was dissolved at a concentration of $100 \mathrm{~g} \mathrm{~L}^{-1}$ with $0.5 \mathrm{M}$ of DTT solution in MOPS buffer (20mM, pH 8) for at least $15 \mathrm{~min}$ at $37^{\circ} \mathrm{C}$ to expose free thiol groups (PMA $\mathrm{Alk}_{\mathrm{SH}}$ ). Subsequently, the polymer solution was diluted with $\mathrm{NaOAc}(50 \mathrm{mM}, \mathrm{pH}$ 4) buffer to a concentration of $1 \mathrm{~g} \mathrm{~L}^{-1}$ just before layering. PVPON was directly dissolved in $\mathrm{NaOAc}$ (50 mM, pH 4) to a concentration of $1 \mathrm{~g} \mathrm{~L}^{-1}$. In a typical experiment, a suspension of $1.16 \mu \mathrm{m}$-diameter $\mathrm{SiO}_{2}$ particles (50 $\mu \mathrm{L}, 5 \mathrm{wt} \%$ suspension) was washed three times with $\mathrm{NaOAc}$ buffer (50 mM, pH 4). $100 \mu \mathrm{L}$ PVPON (1 $\left.\mathrm{g} \mathrm{L}^{-1}\right)$ in NaOAc buffer was added and incubated for $10 \mathrm{~min}$ with constant shaking. The PVPON-coated particles were then washed three times with NaOAc buffer and incubated with $100 \mu \mathrm{L}$ PMA $_{\text {Alk,SH }}\left(1 \mathrm{~g} \mathrm{~L}^{-1}\right)$. The suspension was incubated for $10 \mathrm{~min}$ and washed three times in NaOAc buffer. The outlined procedure describes the assembly of a single polymer bilayer. The adsorption of subsequent interacting polymers (PVPON/PMA ${ }_{\mathrm{Alk}, \mathrm{SH}}$ ) was repeated until 8 bilayers were deposited.

\section{Cross-linking Protocol}

To stabilize the multilayers the particles were exposed to a $2.75 \mathrm{mM}$ solution of chloramine $\mathrm{T}$ (CaT) for 2 min. CaT was first dissolved in Milli-Q water $(0.1 \mathrm{M})$ and subsequently diluted with 2morpholinoethanesulfonic acid (MES) buffer (20 mM, pH 6). The particles were washed twice with MES buffer and twice with NaOAc buffer. Subsequently, the particles were resuspended in $500 \mu \mathrm{L}$ of a bisazide linker $\left(1 \mathrm{~g} \mathrm{~L}^{-1}\right)$, copper(II) sulfate $\left(1.75 \mathrm{~g} \mathrm{~L}^{-1}\right)$, and sodium ascorbate $\left(4.4 \mathrm{~g} \mathrm{~L}^{-1}\right)$ in a 3:1:1 ratio at room temperature overnight. After completion of the multilayer cross-linking, the particles were washed three times with NaOAc buffer and redispersed in $50 \mu \mathrm{L}$ NaOAc buffer. The silica template particles were dissolved with buffered HF (2 M HF/8 M NH${ }_{4} F$ ), and the obtained capsules were washed with at least four washing cycles with NaOAc buffer. (Caution! Hydrofluoric 
acid is highly toxic. Extreme care should be taken when handling HF solution, and only small quantities should be prepared.)

\section{Capsule Labeling}

For visualization, the capsule systems were postlabeled after core template removal to maximize their fluorescence intensity. $50 \mu \mathrm{L}$ of sodium ascorbate $\left(4.4 \mathrm{~g} \mathrm{~L}^{-1}\right), 50 \mu \mathrm{L}$ of copper (II) sulfate $(1.75$ $\left.\mathrm{g} \mathrm{L}^{-1}\right)$, and $1 \mu \mathrm{L}$ of dye $\left(1 \mathrm{~g} \mathrm{~L}^{-1}\right)$ were mixed together. This solution was added to the capsule suspension (starting volume of $50 \mu \mathrm{L}$ of $5 \mathrm{wt} \% 1 \mu \mathrm{m}$-diameter $\mathrm{SiO}_{2}$ particles). The particles were incubated overnight with constant agitation. The fluorescently labeled capsules were then visualized using a fluorescence microscope.

\section{Dextran (Permeability) Studies}

FITC-dextran of five different molar masses (10, 59-77, 250, 500 and $2000 \mathrm{kDa})$ were used for the permeability studies. To investigate the influence of disulfide cross-linking on capsule permeability, equal volumes of the capsule suspension in PBS and FITC-dextran (10 $\mu \mathrm{M}$ at $\mathrm{pH} 7.4$ in PBS) were mixed. The capsules were investigated after 15 min using CLSM. In order to cleave the disulfide linkages, the capsule suspensions were washed three times with $10 \mathrm{mM}$ HEPES, $50 \mathrm{mM} \mathrm{NaCl}$ at $\mathrm{pH}$ 7.2. The samples were then resuspended in $50 \mu \mathrm{L}$ of $5 \mathrm{mM} \mathrm{GSH}$ in $10 \mathrm{mM}$ HEPES, $50 \mathrm{mM} \mathrm{NaCl}$, and incubated in a thermomixer with mild agitation at $37^{\circ} \mathrm{C}$ for 15 minutes, finally being left to cool to room temperature. Equal volumes of the capsule suspensions in HEPES buffer and FITC-dextran (10 $\mu \mathrm{M}$ at $\mathrm{pH} 7.2$ in HEPES) were mixed and observed with CLSM after 15 min.

\section{DNA Hybridization}

In order to obtain double-stranded 45-base pair DNA, two complementary single-stranded 45mer DNA, TXC-AF488 and GXA' were mixed for $1 \mathrm{~h}$ at room temperature under constant agitation in saline sodium citrate buffer. The final concentration of the ds 45 bp DNA was $10 \mu \mathrm{M}$.

\section{DNA Post-encapsulation and Release}

For the DNA permeation studies, the protocol used was identical to that of the dextran permeability studies, where DNA was incubated with “open” capsules for $1 \mathrm{~h}$. Once DNA infiltration was 
confirmed by CLSM, DTDP was added. DTDP was first dissolved in methanol to a concentration of $100 \mathrm{~g} \mathrm{~L}^{-1}$ and then diluted with PBS to a concentration of $0.65 \mathrm{~g} \mathrm{~L}^{-1}$. The mixture of DNA, capsules, and DTDP was left to incubate overnight under constant agitation. Afterwards, the sample was washed three times in PBS buffer to remove any excess DNA and DTDP. DNA encapsulation was observed using CLSM. For the release of DNA, the capsules were once again treated with GSH, as described in the dextran permeability studies. The sample was washed three times with HEPES buffer to remove excess GSH and released DNA, and was investigated with CLSM to confirm the absence of DNA fluorescence.

\section{RESULTS AND DISCUSSION}

\section{Polymer Modification and Characterization}

The key component of this capsule system is a bifunctionalized poly(methacrylic acid) (PMA) that contains two cross-linking sites, which can be applied for the fabrication of dual, covalently stabilized capsules. PMA (15 kDa) was modified in a first step with propargylamine hydrochloride or 2-(pyridylthio)-ethylamine hydrochloride (PDA) (Table S1). The monofunctional PMA was subsequently functionalized with the respective second functional group to form PMA $\mathrm{Alk}_{\mathrm{BDA}}$ (Scheme 1). In this approach four systems containing different functionalization ratios were prepared (Table 1). The complementary polymer, poly( $N$-vinylpyrrolidone) (PVPON, $10 \mathrm{kDa}$ ) was used for layer buildup. This polymer was used without further modification, to allow its removal after capsule preparation.

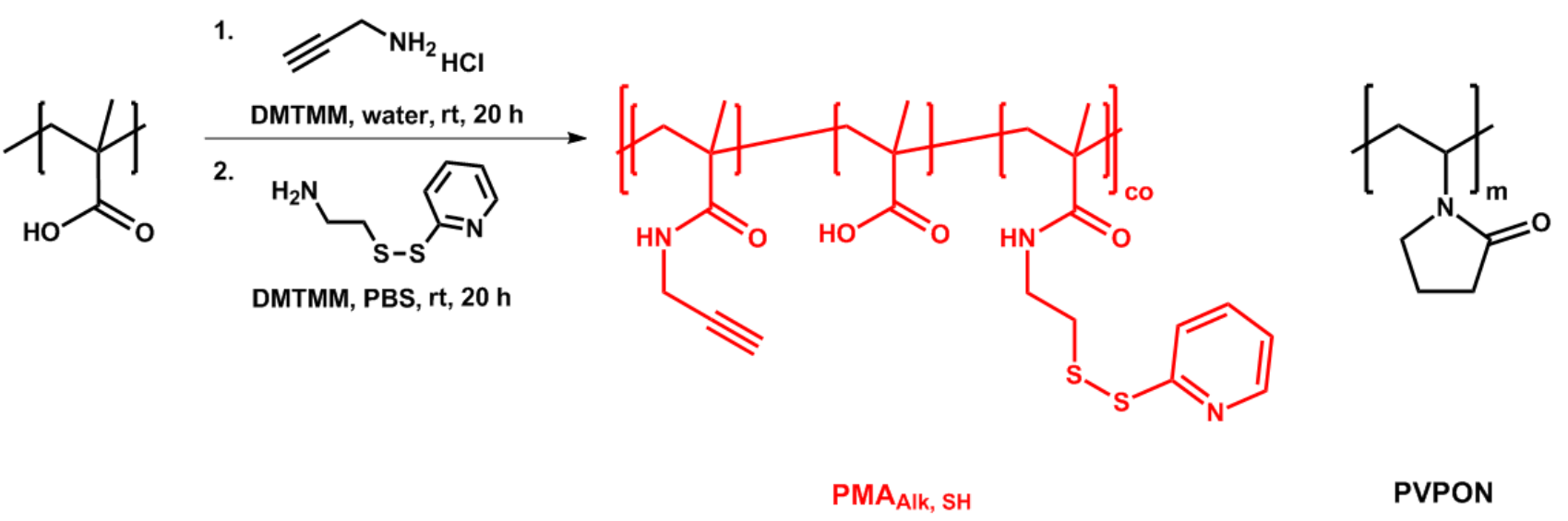


Scheme 1. Schematic representation of the polymer systems used in this study. PMA was modified with amine compounds to introduce alkyne and 2-(pyridylthio)-ethylamine functionalities ( $\left.\mathrm{PMA}_{\mathrm{Alk}, \mathrm{PDA}}\right)$. PVPON was used as received. $\mathrm{rt}=$ room temperature.

The ${ }^{1} \mathrm{H}$ NMR spectra for PMA ${ }_{\text {Alk,PDA_1 }}$ are shown in Fig. 1. The successful reaction with propargylamine hydrochloride is demonstrated by the respective signals, in particular the alkyne proton at about $3.7 \mathrm{ppm}$. The subsequent functionalization with PDA results in the appearance of aromatic signals in the region 7 to $8.5 \mathrm{ppm}$ (corresponding to the pyridyl protecting group) and signals assigned to methylene groups at 2.8 and $3.3 \mathrm{ppm}$ (ethylene spacer). The alkyne and the aromatic signals were used to determine the degree of functionalization (DF; Table 1), respectively.

Table 1. Sample characteristics of bifunctional polymers and nomenclature for the capsules. (DF = degree of functionalization).

\begin{tabular}{lcccccc}
\hline & $\begin{array}{c}\mathbf{D F}_{\mathrm{Alk}}{ }^{\mathbf{a}} \\
{[\%]}\end{array}$ & $\begin{array}{c}\mathbf{D F}_{\mathbf{S H}}{ }^{\mathbf{a}} \\
{[\%]}\end{array}$ & $\begin{array}{c}\mathbf{D F}_{\text {total }}{ }^{\mathbf{a}} \\
{[\%]}\end{array}$ & $\begin{array}{c}\mathbf{M}_{\mathbf{n}}{ }^{\mathbf{b}} \\
{\left[\mathrm{g} \mathrm{mol}^{-1}\right]}\end{array}$ & PDI $^{\mathbf{b}}$ & $\begin{array}{c}\text { Capsule } \\
\text { description }\end{array}$ \\
\hline PMA Alk,PDA_1 & 7 & 5 & 12 & 35,600 & 1.9 & $\mathbf{C 0}$ \\
PMA Alk,PDA_2 & 14 & 5 & 19 & 38,100 & 2.1 & $\mathbf{C 1}$ \\
PMA & 12 & 11 & 23 & 36,000 & 1.9 & $\mathbf{C 2}$ \\
PMAlk,PDA_3 $_{\text {Alk,PDA_4 }}$ & 9 & 16 & 25 & 40,400 & 1.8 & $\mathbf{C 3}$ \\
\hline
\end{tabular}

${ }^{\text {a }}$ determined by ${ }^{1} \mathrm{H}$ NMR $\left(\mathrm{D}_{2} \mathrm{O}, 400 \mathrm{MHz}\right) ;{ }^{\text {b }}$ determined by SEC (eluent: water) 


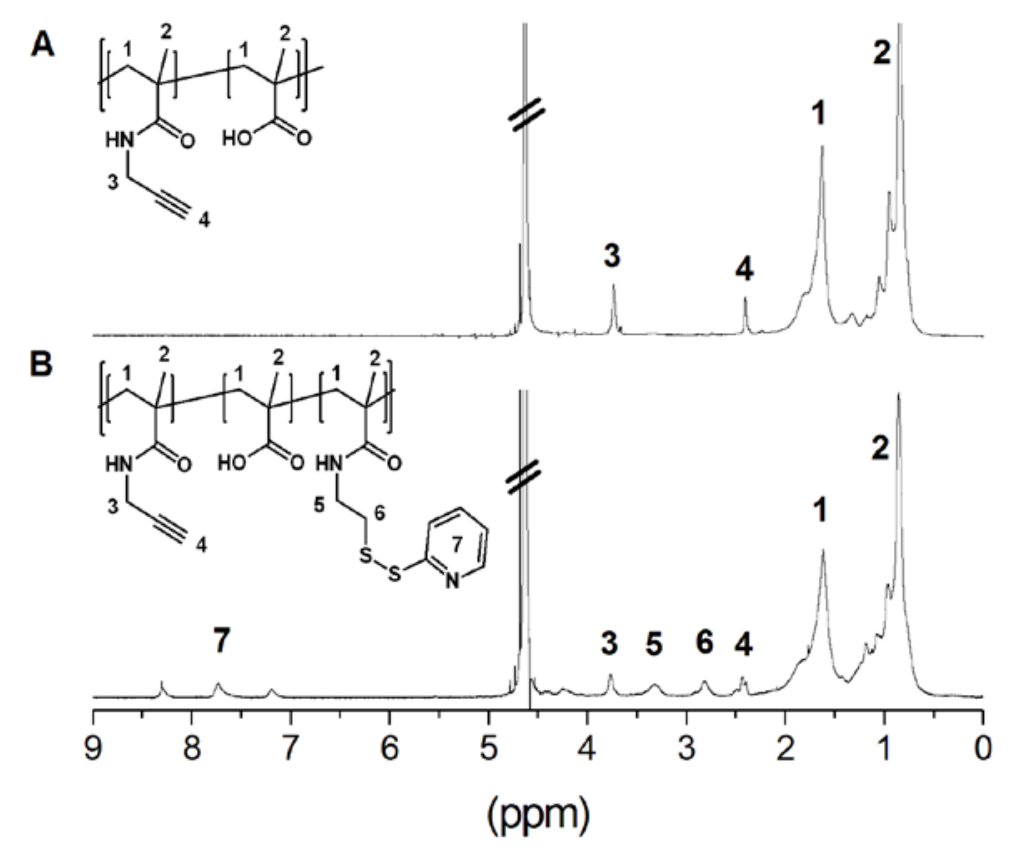

Fig. 1. ${ }^{1} \mathrm{H}$ NMR spectra $\left(\mathrm{D}_{2} \mathrm{O}, 400 \mathrm{MHz}\right)$ of the monofunctional $\mathrm{PMA}_{\mathrm{Alk} \_} 1(\mathrm{~A})$ and the bifunctional $\mathrm{PMA}_{\mathrm{Alk}, \text { PDA_1 }}(\mathrm{B})$.

According to size exclusion chromatography (SEC) measurements, the polymers used were polydisperse (Table 1, S1). A shift to higher molar masses after monofunctionalization was observed. The introduction of the second functionality caused a further increase in the polydispersity index (PDI) value and the occurrence of bimodal SEC traces.

\section{Multilayer Assembly on Particles}

The multilayer films were assembled based on hydrogen bonding interactions between the hydrogen donor PMA and the acceptor PVPON to form (PVPON/PMA $A_{\text {Alk,SH}}$ ) multilayers (Scheme 2). Prior to LbL assembly, PMA Alk,PDA was exposed to a dithiothreitol (DTT) solution to generate free thiol groups $\left(\mathrm{PMA}_{\mathrm{Alk}, \mathrm{SH}}\right)$. Silica particles were sequentially exposed to PVPON and PMA $\mathrm{Alk}_{\mathrm{SH}}$ solutions (1 $\mathrm{g} \mathrm{L}^{-1}$; 50 mM sodium acetate, $\mathrm{pH}$ 4) for 10 min, respectively. 

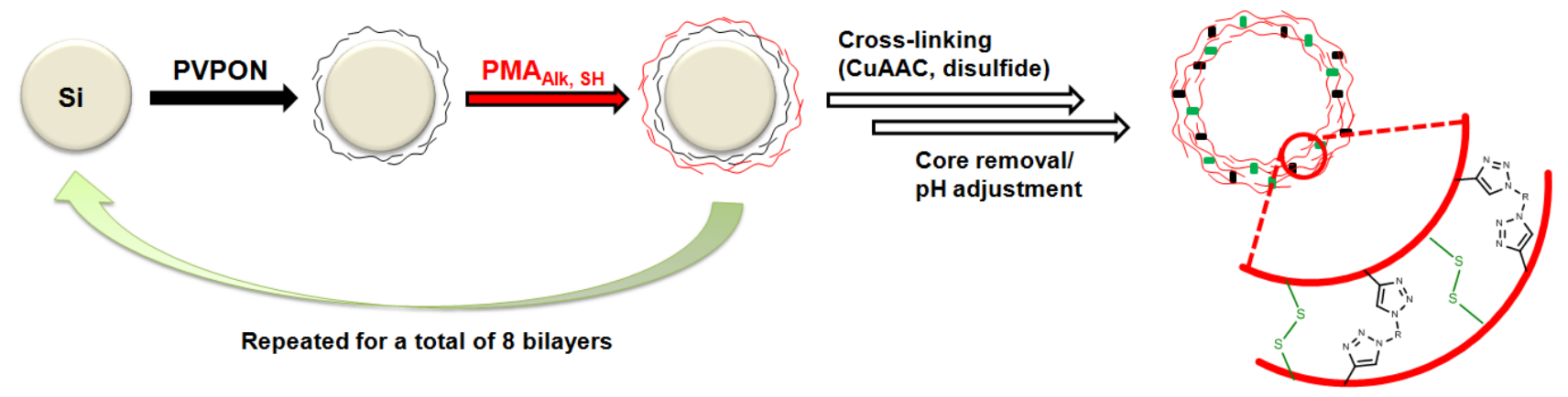

Scheme 2. Assembly of (PVPON/PMA $\left.\mathrm{Alk}_{\mathrm{SH}}\right)_{8}$ multilayers. PVPON was deposited on sacrificial silica particle templates, followed by $\mathrm{PMA}_{\mathrm{Alk}, \mathrm{SH}}$. This process was repeated until 8 bilayers were deposited. Both crosslinking steps were carried out prior to the removal of the sacrificial core with buffered hydrofluoric acid.

To quantify the multilayer buildup, PVPON was functionalized with the dye AF488-NHS, and the fluorescence of the core-shell particles was measured by flow cytometry (Fig. 2). Monofunctional $\mathrm{PMA}_{\mathrm{Alk} \_2}$ and bifunctional PMA $\mathrm{Alk}, \mathrm{SH} \_2_{2}$ were compared to study their respective influence on layer buildup. In both cases, the buildup of the multilayers was regular and linear, with the dualfunctionalized system giving slightly higher values. At 8-bilayers the adsorbed amount of PMA $_{\text {Alk,SH_2 }}$ was about $30 \%$ more than for PMA Alk_2 $_{2}$ A similar behavior was observed when comparing the buildup of PMA/PVPON and PMA $\mathrm{SH}_{\mathrm{S}} / \mathrm{PVPON}$, and the difference was attributed to a greater mass per hydrogen-bonded group. ${ }^{28}$

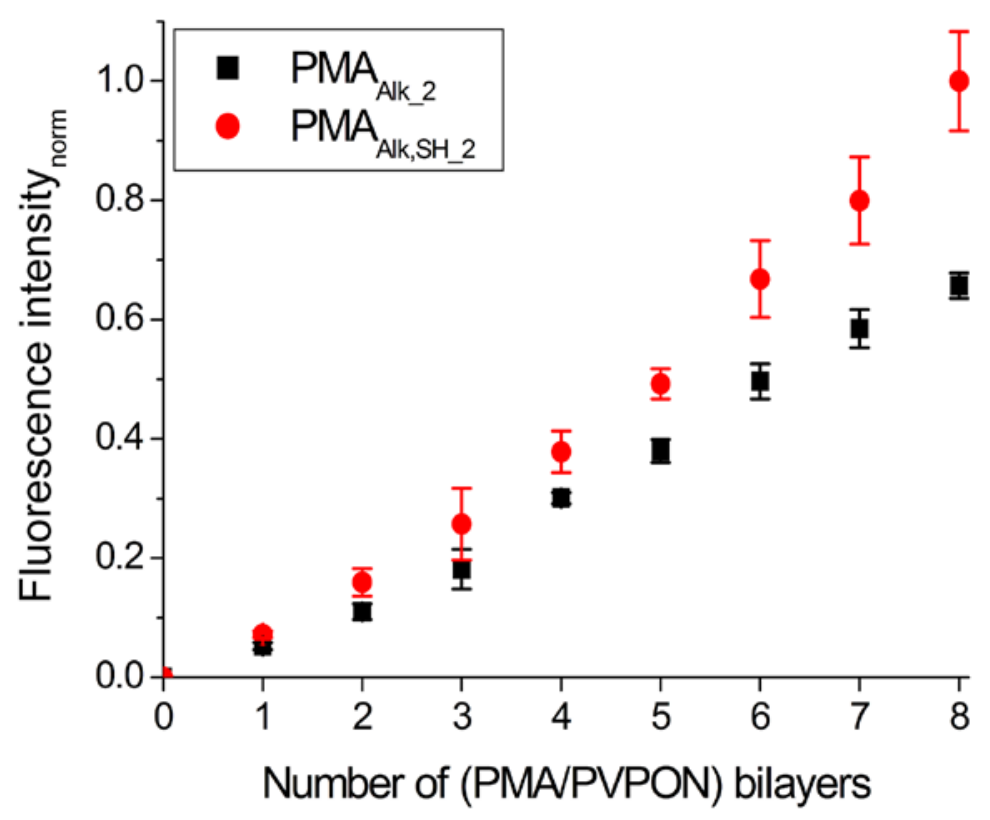


Fig. 2. Normalized fluorescence of $1 \mu$ m-diameter $\mathrm{SiO}_{2}$ particles coated with PVPON/PMA multilayers, as measured by flow cytometry. PVPON was labeled with AF488-NHS. The multilayers were deposited from 1 $\mathrm{g} \mathrm{L}^{-1}$ polymer solutions (50 mM NaOAc, $\mathrm{pH}$ 4) and measurements were taken after each PVPON layer was deposited. Layer 0 corresponds to the first PVPON layer deposited.

\section{Capsule Assembly}

To stabilize the multilayer films obtained, disulfide and CuAAC cross-linking reactions were performed. For the latter, following the assembly of all layers, a non-degradable bisazide crosslinker was applied to stabilize the films. The CuAAC cross-linking was carried out using $\mathrm{CuSO}_{4}$ (1.75 $\left.\mathrm{g} \mathrm{L}^{-1}\right)$ and sodium ascorbate $\left(4.4 \mathrm{~g} \mathrm{~L}^{-1}\right)$. The bilayer films were incubated overnight with the bisazide cross-linker. Disulfide stabilization was achieved by exposing the particles to chloramine $\mathrm{T}$ (CaT) solution for $1 \mathrm{~min}$, followed by several washing steps. Finally, capsules were obtained by removal of the silica particle templates using $5 \mathrm{M}$ hydrogen fluoride (HF)/ammonium fluoride buffered to $\mathrm{pH} 5$.

In a first attempt (data not shown), capsules $(\mathrm{C} 0-\mathrm{C} 3$, Table 1$)$ were prepared of all bifunctional $\mathrm{PMA}_{\text {Alk,PDA}}$. The successful synthesis of stabilized capsules was observed by fluorescence microscopy. To obtain information on the influence of the degree of functionalization on the capsule properties, the capsules were investigated in the "open” state as well. Whereas the loss of the disulfide stabilization did not affect C1, C2 and C3 capsules, C0 capsules lost their integrity. Almost all C0 capsules obtained showed a highly deformed shape. Clearly, 7\% alkyne functionalization is not sufficient to stabilize the capsules without the co-stabilization effect of the disulfide bonds. Therefore, further studies were performed solely using $\mathrm{C} 0-\mathrm{C} 3$ capsules.

Fluorescence microscopy and atomic force microscopy (AFM) images of the capsules prepared from $\mathrm{PMA}_{\mathrm{Alk}, \mathrm{SH} \_2}$ and $\mathrm{PMA}_{\mathrm{Alk} \_2}$ (see below) are shown in Fig. 3. For visualization, the shell of the capsules was labeled with AF488- $\mathrm{N}_{3}$. Both systems showed a spherical shape for the capsules formed (Fig. 3A). AFM analysis of the air-dried capsules revealed spherical structures with creases 
for the $\mathrm{PMA}_{\mathrm{Alk} \_2}$ capsules (Fig. 3B inset). However, PMA $\mathrm{Alk}, \mathrm{SH} \_2_{2}$ capsules exhibited oval shapes with folds in the center (Fig. 3B). Noteworthy, the capsules differed significantly in their wall thickness, as demonstrated by cross-sectional image profiles (Fig. S1). Analysis of these data provided a wall thickness of $0.8 \pm 0.1 \mathrm{~nm}$ and $2.3 \pm 0.1 \mathrm{~nm}$ per $\mathrm{PMA}_{\mathrm{Alk}}$ and $\mathrm{PMA}_{\mathrm{Alk}, \mathrm{SH}}$ layer, respectively. This finding supports the observation of a higher mass adsorption for $\mathrm{PMA}_{\mathrm{Alk}, \mathrm{SH}}$ (as shown above by flow cytometry study (Fig. 2)), leading to a larger layer thickness..
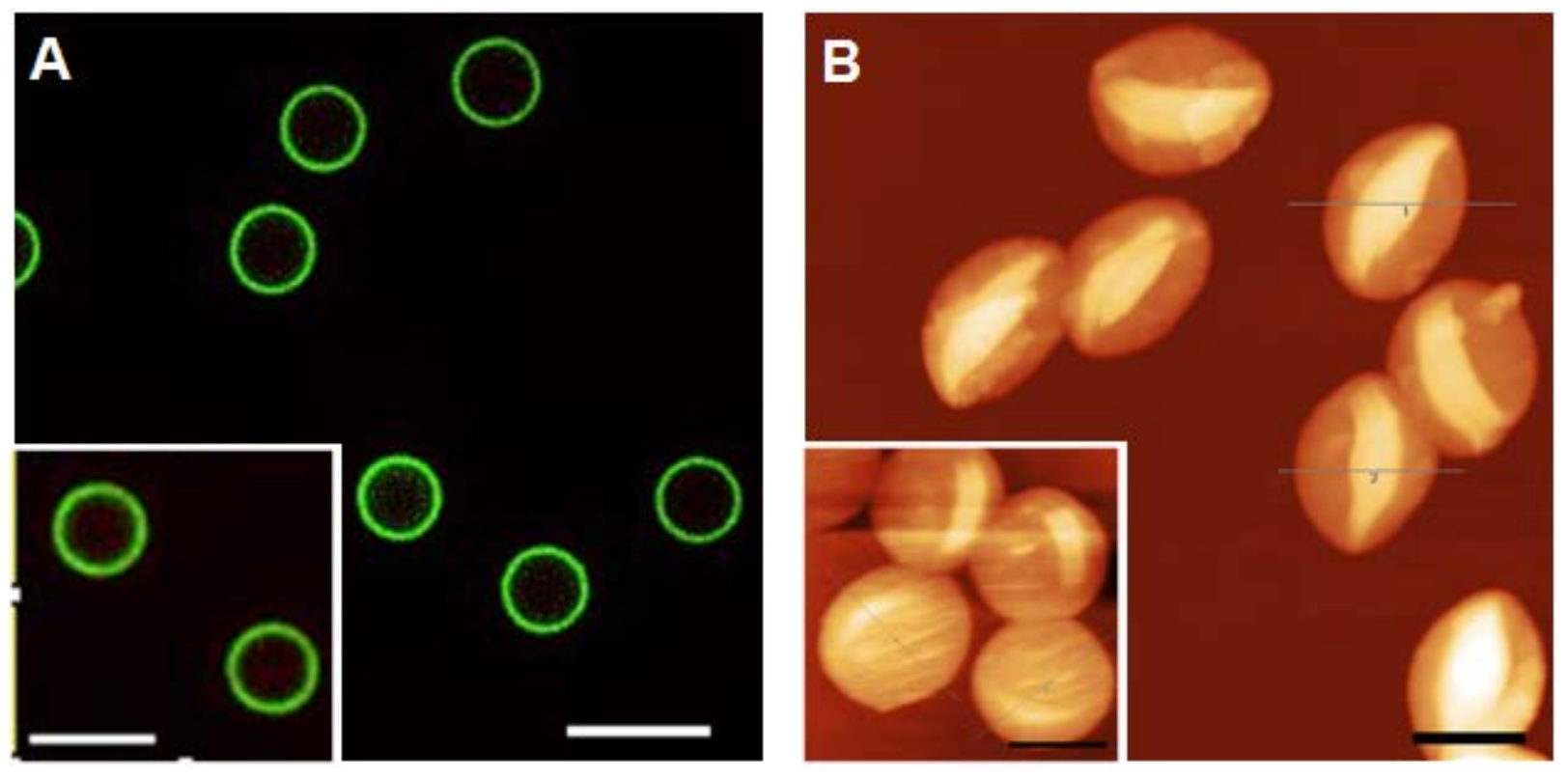

Fig. 3. Fluorescence microscopy (A) and atomic force microscopy (B) images of capsules obtained from $\mathrm{PMA}_{\mathrm{Alk}, \mathrm{PDA} \_2}\left(\mathrm{C} 1\right.$ capsules) and $\mathrm{PMA}_{\text {Alk }}$ (inset). The shell of the capsules was labeled with AF488- $\mathrm{N}_{3}$. Scale bar $=2 \mu \mathrm{m}$.

\section{Capsule pH Responsiveness}

The carboxyl groups of PMA allow the polymer to respond to external stimuli. With a $\mathrm{pK}_{\mathrm{a}}$ of 5.6, the unsubstituted PMA exists in the protonated state at low $\mathrm{pH}$, whereas at physiological $\mathrm{pH}$ or higher mostly deprotonated carboxylic acid groups are present. The latter case (high $\mathrm{pH}$ ) causes electrostatic repulsion between the charged groups, which can lead to swelling in a cross-linked network. The responsiveness of the capsules prepared from $\mathrm{C} 1-\mathrm{C} 3$ was examined by exposing the 
capsules to NaOAc buffer ( $\mathrm{pH}$ 4) and $\mathrm{PBS}(\mathrm{pH} 7.4)$, constituting one $\mathrm{pH}$ cycle. To prove the reversibility of the capsule swelling, three $\mathrm{pH}$ cycles were performed (Fig. 4).

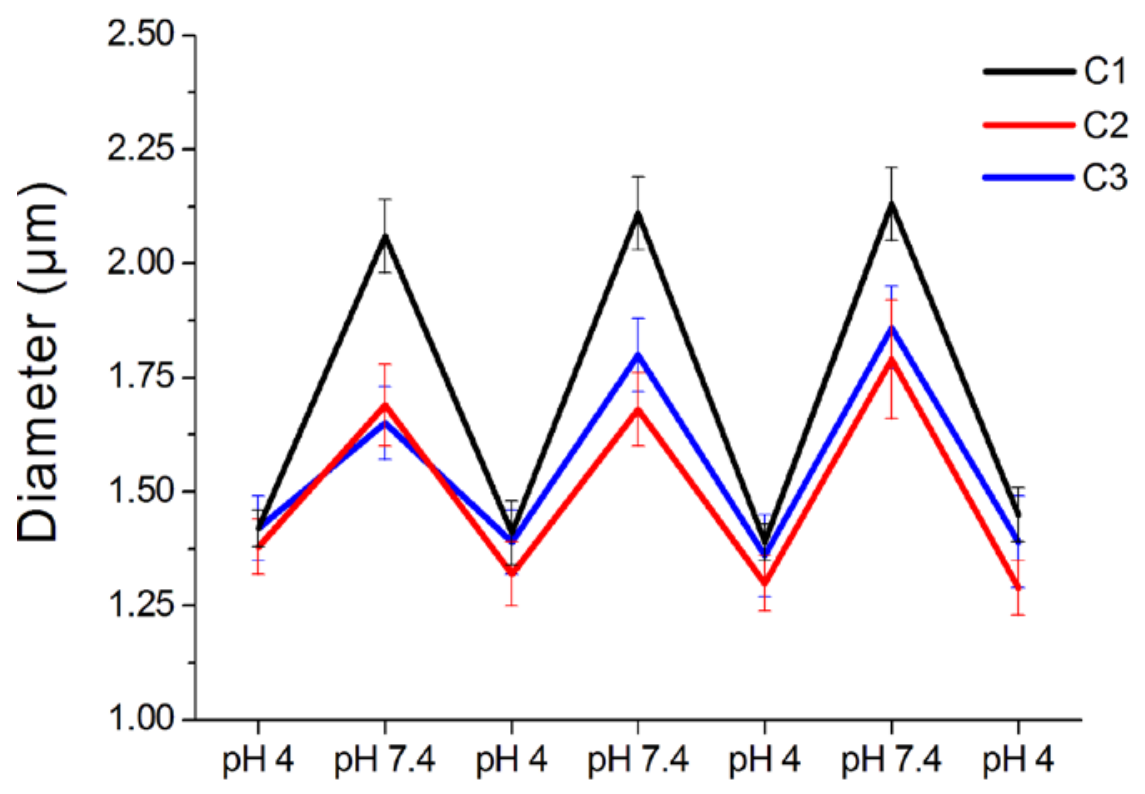

Fig. 4. $\mathrm{pH}$ responsiveness of $\left(\mathrm{PMA}_{\mathrm{Alk}, \mathrm{SH}}\right)_{8}(\mathrm{C} 1, \mathrm{C} 2, \mathrm{C} 3)$ capsules. AF647-labeled capsules were cycled through pH 4 and pH 7.4 solutions, and capsule diameters were measured from fluorescence microscopy images. Data were averaged from at least 30 images. Error bars show the standard deviation of capsule diameters.

The size of the capsules was determined by fluorescence microscopy, averaged over a population of at least 30 capsules. The capsules were post-labeled with AF647-maleimide. The pH value was changed subsequently by washing the capsules with the respective buffer solutions. In all cases, an increase in the capsule diameter was observed upon exposing them to $\mathrm{pH}$ 7.4. However, the degree of PMA functionalization as well as the capsule preparation technique influences the swelling behavior. C1 capsules exhibit the most significant difference in terms of swelling, due to the lowest total degree of functionalization and thus cross-linking. The increase of disulfide-to-CuAAC crosslinking ratio resulted in capsules with a much lower degree of swelling, thus, demonstrating more efficient cross-linking. This effect might be attributed to the shorter distance between the individual polymer chains upon disulfide cross-linking. In contrast, the bisazide PEG linker used for the 
CuAAC cross-linking consists of eight ethylene glycol repeating units, which suggests polymer chains can be separated further apart.

By switching the capsules from the "closed" to the "open" state, the disulfide bonds are severed and the respective stabilization is disrupted. Thus, similar $\mathrm{pH}$ studies should demonstrate a higher degree of swelling when adjusted to $\mathrm{pH} 7.4$ (Table 2). In the "open” state, CuAAC cross-links alone exert less capsule stability and the electrostatic repulsion of the partly deprotonated carboxylic acids at higher $\mathrm{pH}$ is more pronounced. The difference between the swelling in the "open" and "closed" state is in a similar range for $\mathrm{C} 1$ to $\mathrm{C} 3$, even though a stronger increase was expected for systems with higher disulfide linkages. Unexpected values were obtained for C3 capsules. The swelling is less pronounced, showing almost no difference between the "closed" and "open" state. The polymer precursor exhibits the highest overall functionalization and highest percentage of disulfide crosslinking moieties. Comparable swelling behavior in the "closed" state and the low swelling difference upon "opening" demonstrate that higher functionalization is not necessary. The negative impact of a too high functionalization on the capsule stability was also reported for thiol-modified (PMA $\mathrm{SH}_{\mathrm{H}}$ capsules. $^{36}$

Table 2. Percentage swelling (\%) of capsules when increasing $\mathrm{pH}$ from 4 to 7.4 . Capsule diameters were determined by fluorescence microcopy.

\begin{tabular}{cccc}
\hline Capsules & DF $_{\text {total }}{ }^{\text {a }}$ [\%] & \multicolumn{2}{c}{ Swelling [\%] } \\
“Closed” state “Open” state
\end{tabular}

${ }^{\mathrm{a}}$ degree of functionalization determined by ${ }^{1} \mathrm{H}$ NMR $\left(\mathrm{D}_{2} \mathrm{O}, 400 \mathrm{MHz}\right)$

\section{Capsule Permeability Studies}

The permeability of the capsules was investigated using confocal laser scanning microscopy (CLSM). A range of sizes of fluorescently labeled dextrans (FITC-dextran) were incubated with the 
capsules at $\mathrm{pH} 7.4$ (PBS buffer), and after 15 min of incubation the samples were investigated by CLSM. All samples were measured in both the "closed" and "open" states in order to study the permeability of the capsules and their ability to switch from "closed" to "open" and vice versa. The reversible reaction of disulfides to thiols has been well documented for controlling the structure of proteins in a biological environment. ${ }^{37}$ Recently, it has also gained attention in designing bioresponsive materials, particularly delivery systems, as it allows for specific release of cargo in cellular environments. Previous studies on disulfide-functionalized capsules show that disulfide bonds can be cleaved in a simulated cellular environment using reagents such as glutathione (GSH). ${ }^{28}$ Due to the loss of cross-linking, such capsules degraded completely following disulfide cleavage. In contrast, the capsules synthesized in the present study are dual cross-linked, enabling them to maintain their integrity, even when the disulfide bonds are cleaved. Consequently, in this system, the disulfide functionality can be used to reversibly alter the permeability properties of the capsules. To this end, the capsules were treated with glutathione, washed with buffer solution and incubated with the respective FITC-dextran.

All capsules except C3 capsules were impermeable to 2000, 500 and $250 \mathrm{kDa}$ dextran; independent of the capsules state, as can be seen from the CLSM images (Fig. S2 and S3). Furthermore, all samples were permeable to $10 \mathrm{kDa}$ dextran in the "closed" state, whereas the $59-77 \mathrm{kDa}$ dextran represents a borderline case (Fig. S2). Both, C1 and C2 capsules were impermeable to this dextran size in the "closed" state, with having minor number of dextran molecules penetrating into the capsules. However, in the "open" state after reduction of the disulfide bonds, C2 capsules become permeable to the 59-77 kDa dextran (Fig. 5). The cleavage of a higher number of disulfide crosslinks causes a decrease in cross-linking density, which is sufficient to switch the permeability of the system. In contrast, the difference in cross-linking between the "closed" and "opened" state for C1 capsules is not sufficient to significantly change the permeability (Fig. S3). As already observed in the $\mathrm{pH}$ studies, C3 capsules do not seem to have optimal cross-linking. Already in the "closed" state, high molar mass dextran permeates into the capsule interior, as can be seen by the fluorescent core 
of the capsules. Therefore, these capsules were excluded from subsequent DNA encapsulation studies.
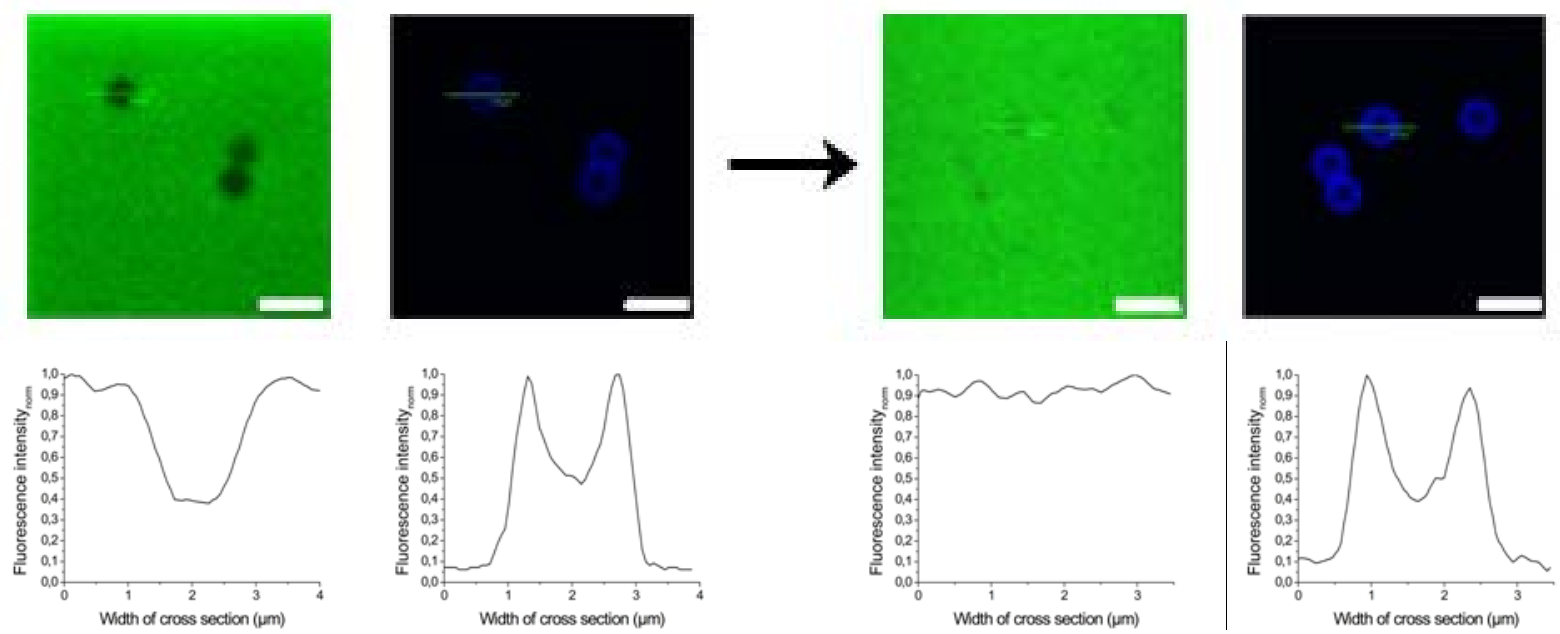

Fig. 5. CLSM images of C2 capsules incubated with FITC-dextran (59-77 kDa) before ("closed" state; left) and after ("open" state; right) cleavage of disulfide bonds with GSH. The capsule wall was labeled with Alexa Fluor 647 (blue emission). Representative cross-sectional fluorescence profiles for each image are shown below the images. All scale bars represent $3 \mu \mathrm{m}$.

\section{DNA Encapsulation and Release Studies}

To qualitatively investigate the potential of these systems for the encapsulation and release of biorelevant cargo, the reversible nature of the disulfide bond was exploited. The capsules were incubated in conditions tailored to the reduction of the disulfide groups (with GSH) and crosslinking them again. The latter was accomplished using the dynamic pyridyl-thiol-exchange reaction. ${ }^{8,}{ }^{36}$ Formation of disulfide bonds through oxidation was unsuitable, as the harsh conditions can lead to oxidative damage of DNA and the dye labels, which renders it unusable. 45 bp dsDNA was selected as model cargo due to its similar molar mass to the above tested dextrans (59-77 $\mathrm{kDa})$, for which the systems showed promising tunable permeability. It should be taken into consideration that DNA and dextran have different physical properties, where the former exhibits higher rigidity and negative charge, making it difficult to directly compare results obtained in both studies. In order to encapsulate DNA, "open" state type capsules C1 and C2 were incubated with labeled DNA. 
CLSM measurements were performed after $15 \mathrm{~min}$ and $1 \mathrm{~h}$ to check the time dependent permeation. 2,2'-dithiodipyridine (DTDP) was added to reestablish the disulfide bonds, effectively switching the capsules to the "closed" state and encapsulating the DNA. DTDP selectively attacks thiol functionalities and does not affect other labile groups or functionalities. This dynamic exchange reaction is considered to be slower than the typical oxidation reaction with CaT, hence the capsules were exposed to the DTDP solution for $21 \mathrm{~h}$. Using DTDP instead of CaT had two distinct advantages. Firstly, DTDP does not induce oxidative damage to any component of the experiment, especially DNA and the fluorescent dye. Moreover, as both the capsule wall and DNA possess negative charge, the prolonged incubation time might improve DNA loading. CLSM images were taken after washing the "closed" capsules to remove background fluorescent DNA. In the last step, the disulfide bonds were “opened” again to release the DNA (Scheme 3).

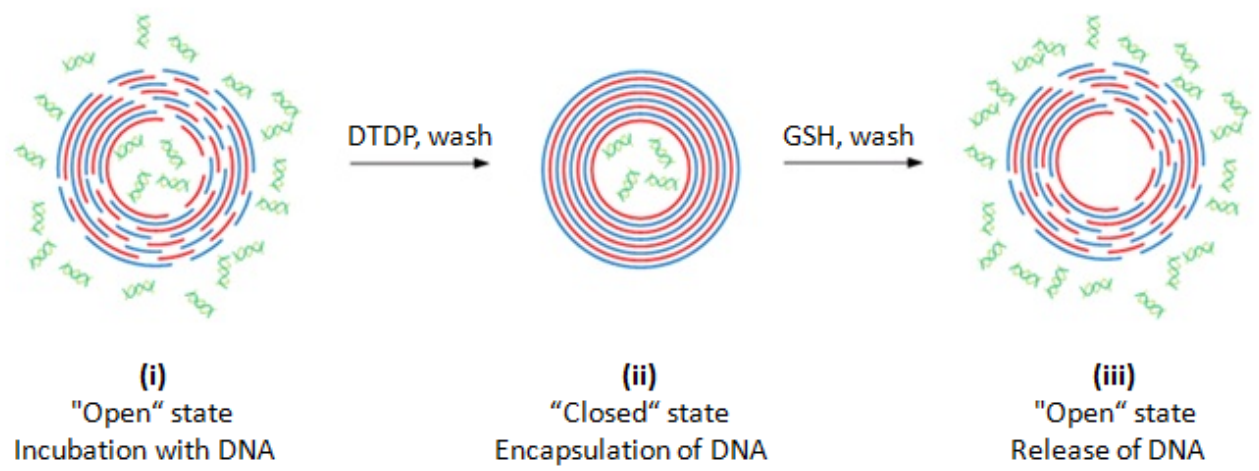

Scheme 3. Schematic representation of DNA encapsulation and release. Capsules in the "open" state are incubated with labeled DNA, which allows the DNA to permeate into capsules (i). The capsules are treated with 2,2'-dithiodipyridine (DTDP) to switch them to the "closed” state, effectively encapsulating DNA within (ii). To switch the capsules back to the "open" state, the capsules are treated with glutathione (GSH), allowing DNA to diffuse out of capsules, effectively releasing DNA.

Fig. 6 shows the results obtained for C1 capsules. "Open" state capsules were incubated with labeled DNA. CLSM images demonstrate high permeability for DNA. This finding was further supported by images taken after reestablishing the disulfide bonds. The DNA, which diffused into the capsules remained, and thus was encapsulated, as indicated by the fluorescence images at $488 \mathrm{~nm}$. After 
exposing the capsules to a GSH solution the DNA was released again, as demonstrated by the disappearance of the fluorescence in the respective CLSM images. The C1 capsules appear to be the better system for DNA encapsulation in this study compared to the C2 capsules, which appeared to be the optimal system in the permeability studies with dextran. Fluorescently labeled DNA was in particular entrapped within the capsule walls of C2 capsules, which bear a higher number of thiol functionalities (Fig. S4). This observation might be an indication of strong interaction of the DNA with the capsule walls during the "closing procedure", demonstrating the drawback of the postloading approach in comparison to pre-loading approaches. Furthermore, a closer inspection of a set of images revealed the existence of residual fluorescence of DNA within the capsule walls (data not shown). After an extended period of time during which the capsules were kept in washing solution, the fluorescence disappeared completely. However, it should be considered that cross-linking efficiencies differ for CaT and DTDP triggered cross-linking, ${ }^{36}$ which might have an effect on the permeability of the system and further explains the differences observed for capsules C1 and C2 in the permeability studies with dextrans and the encapsulation study with DNA.

\section{CONCLUSIONS}

We have reported the synthesis of bifunctional poly(methacrylic acid) (PMA) for the preparation of LbL capsule systems with reversible permeability. These capsules were assembled based on hydrogen-bonding. The dual stabilization of the capsules with two different kinds of cross-linkers, of which one was a reducible linker, allowed the design of gateable systems. The permeability of the capsules could be tuned by switching the disulfide groups to thiol moieties and vice versa. The amount of cross-linking and swelling can be controlled by the degree of functionalization of the polymer. Switching from the "closed" (both cross-linkers intact) to the "open" state (only CuAAc) was evident from permeability studies with different-sized dextrans. Furthermore, the potential of the system to reversibly encapsulate DNA cargo was demonstrated. This study provides first insights into a new generation of capsules for potential use in a range of therapeutic and diagnostic applications. The gated porosity might find application in loading cargo, such as biomolecules, and 
their subsequent specific release in cellular environments. In addition, as the capsules remain intact, the capsules could also be used as microreactors. Extension of the approach presented in this study to other polymer systems as well as other dual stabilization methods are currently under investigation.
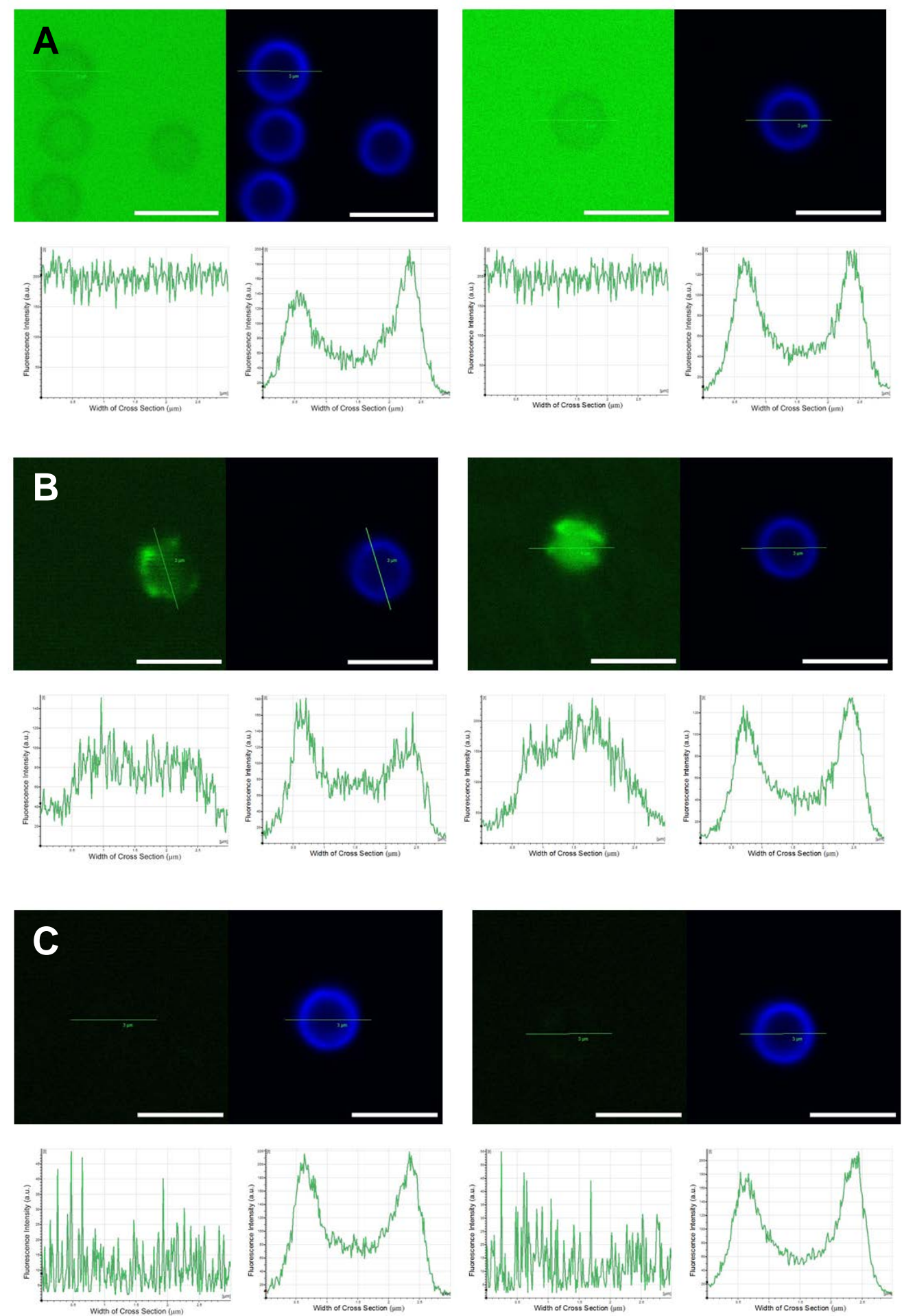

Fig. 6. Encapsulation and release study of C1 capsules with 45 bp ds DNA using CLSM (scale bar $=2 \mu \mathrm{m}$ ). A: Incubation of capsules in the "open" state after 15 min (left) and $1 \mathrm{~h}$ (right). B: Encapsulation of DNA; 
capsules were switched to the "closed" state, followed by three washing steps. C: Release of DNA; disulfide bonds were cleaved, switching the capsules to the "open" state again.

\section{ASSOCIATED CONTENT}

Supporting Information. Sample characteristics of monofunctional polymers, cross-sectional AFM

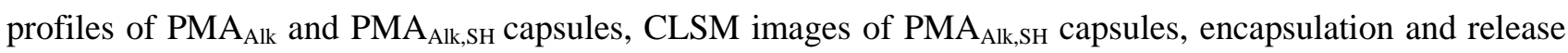
study of C2 capsules using CLSM. This material is available free of charge via the Internet at http://www.sciencedirect.com.

\section{AUTHOR INFORMATION}

\section{Corresponding Author}

*Email: fcaruso@unimelb.edu.au

Tel.: +61383443461

\section{Notes}

The authors declare no competing financial interest.

\section{Acknowledgments}

K.K. is grateful to the Alexander von Humboldt-Foundation (Feodor-Lynen fellowship) for financial support. M.M. acknowledges The University of Melbourne for a McKenzie Fellowship. F.C. acknowledges the Australian Research Council for funding under the Australian Laureate Fellowship scheme (FL1201100030). Dr. Juan Mendez and Dr. Georgina Such are thanked for initial studies and discussions on the development of capsules with gated permeability.

\section{References}

1. van Dongen, S. F. M.; de Hoog, H. P. M.; Peters, R. J. R. W.; Nallani, M.; Nolte, R. J. M.; van Hest, J. C. M., Chem. Rev. 2009, 109, 6212-6274.

2. $\quad$ De Koker, S.; Hoogenboom, R.; De Geest, B. G., Chem. Soc. Rev. 2012, 41, 2867-2884.

3. Abulateefeh, S. R.; Spain, S. G.; Aylott, J. W.; Chan, W. C.; Garnett, M. C.; Alexander, C., Macromol. Biosci. 2011, 11, 1722-1734.

4. $\quad$ Decher, G.; Hong, J. D.; Schmitt, J., Thin Solid Films 1992, 210, 831-835.

5. $\quad$ Decher, G., Science 1997, 277, 1232-1237.

6. $\quad$ Caruso, F.; Caruso, R. A.; Mohwald, H., Science 1998, 282, 1111-1114.

7. $\quad$ Kreft, O.; Georgieva, R.; Bäumler, H.; Steup, M.; Müller-Röber, B.; Sukhorukov, G. B.; Möhwald, H., Macromol. Rapid Commun. 2006, 27, 435-440.

8. Sivakumar, S.; Bansal, V.; Cortez, C.; Chong, S. F.; Zelikin, A. N.; Caruso, F., Adv. Mater. 2009, 21, 1820-1824.

9. Z Zhang, X.; Chen, H.; Zhang, H. Y., Chem. Commun. 2007, 1395-1405.

10. $\quad$ Peyratout, C. S.; Dahne, L., Angew. Chem. Int. Ed. 2004, 43, 3762-3783. 
11. Such, G. K.; Johnston, A. P. R.; Caruso, F., Chem. Soc. Rev. 2011, 40, 19-29.

12. Bergbreiter, D. E.; Liao, K. S., Soft Matter 2009, 5, 23-28.

13. Becker, A. L.; Johnston, A. P. R.; Caruso, F., Small 2010, 6, 1836-1852.

14. Johnston, A. P. R.; Such, G. K.; Ng, S. L.; Caruso, F., Curr. Opin. Colloid Interface Sci. 2011, 16, 171-181.

15. De Cock, L. J.; De Koker, S.; De Geest, B. G.; Grooten, J.; Vervaet, C.; Remon, J. P.; Sukhorukov, G. B.; Antipina, M. N., Angew. Chem. Int. Ed. 2010, 49, 6954-6973.

16. Ma, Y. J.; Dong, W. F.; Hempenius, M. A.; Mohwald, H.; Vancso, G. J., Nat. Mater. 2006, 5, 724-729.

17. Ibarz, G.; Dahne, L.; Donath, E.; Mohwald, H., Adv. Mater. 2001, 13, 1324-1327.

18. Wang, A. H.; Tao, C.; Cui, Y.; Duan, L.; Yang, Y.; Li, J. B., J. Colloid Interf. Sci. 2009, 332, 271-279.

19. Huang, C. J.; Chang, F. C., Macromolecules 2009, 42, 5155-5166.

20. Sukhorukov, G. B.; Antipov, A. A.; Voigt, A.; Donath, E.; Mohwald, H., Macromol. Rapid Commun. 2001, 22, 44-46.

21. Kozlovskaya, V.; Wang, Y.; Higgins, W.; Chen, J.; Chen, Y.; Kharlampieva, E., Soft Matter 2012, 8, 9828-9839.

22. Kozlovskaya, V.; Chen, J.; Tedjo, C.; Liang, X.; Campos-Gomez, J.; Oh, J.; Saeed, M.; Lungu, C. T.; Kharlampieva, E., J. Mater. Chem. B 2014, 2, 2494-2507.

23. Tong, W. J.; Gao, C. Y.; Mohwald, H., Macromolecules 2006, 39, 335-340.

24. Cheng, R.; Feng, F.; Meng, F. H.; Deng, C.; Feijen, J.; Zhong, Z. Y., J. Control Release 2011, 152, 2-12.

25. Zelikin, A. N.; Li, Q.; Caruso, F., Chem. Mater. 2008, 20, 2655-2661.

26. Zelikin, A. N.; Becker, A. L.; Johnston, A. P. R.; Wark, K. L.; Turatti, F.; Caruso, F., ACS Nano 2007, 1, 63-69.

27. Sexton, A.; Whitney, P. G.; Chong, S. F.; Zelikin, A. N.; Johnston, A. P. R.; De Rose, R.; Brooks, A. G.; Caruso, F.; Kent, S. J., ACS Nano 2009, 3, 3391-3400.

28. Zelikin, A. N.; Quinn, J. F.; Caruso, F., Biomacromolecules 2006, 7, 27-30.

29. Meldal, M.; Tornoe, C. W., Chem. Rev. 2008, 108, 2952-3015.

30. Lutz, J. F.; Zarafshani, Z., Adv. Drug Deliv. Rev. 2008, 60, 958-970.

31. Golas, P. L.; Matyjaszewski, K., Chem. Soc. Rev. 2010, 39, 1338-1354.

32. Kempe, K.; Krieg, A.; Becer, C. R.; Schubert, U. S., Chem. Soc. Rev. 2012, 41, 176-191.

33. Kolb, H. C.; Finn, M. G.; Sharpless, K. B., Angew. Chem. Int. Ed. 2001, 40, 2004-2021.

34. Such, G. K.; Gunawan, S. T.; Liang, K.; Caruso, F., Macromol. Rapid Commun. 2013, 34, 894-902.

35. Lee, L.; Johnston, A. P. R.; Caruso, F., Langmuir 2012, 28, 12527-12535.

36. $\quad$ Chong, S. F.; Lee, J. H.; Zelikin, A. N.; Caruso, F., Langmuir 2011, 27, 1724-1730.

37. Bauhuber, S.; Hozsa, C.; Breunig, M.; Gopferich, A., Adv. Mater. 2009, 21, 3286-3306. 
Graphic Abstract

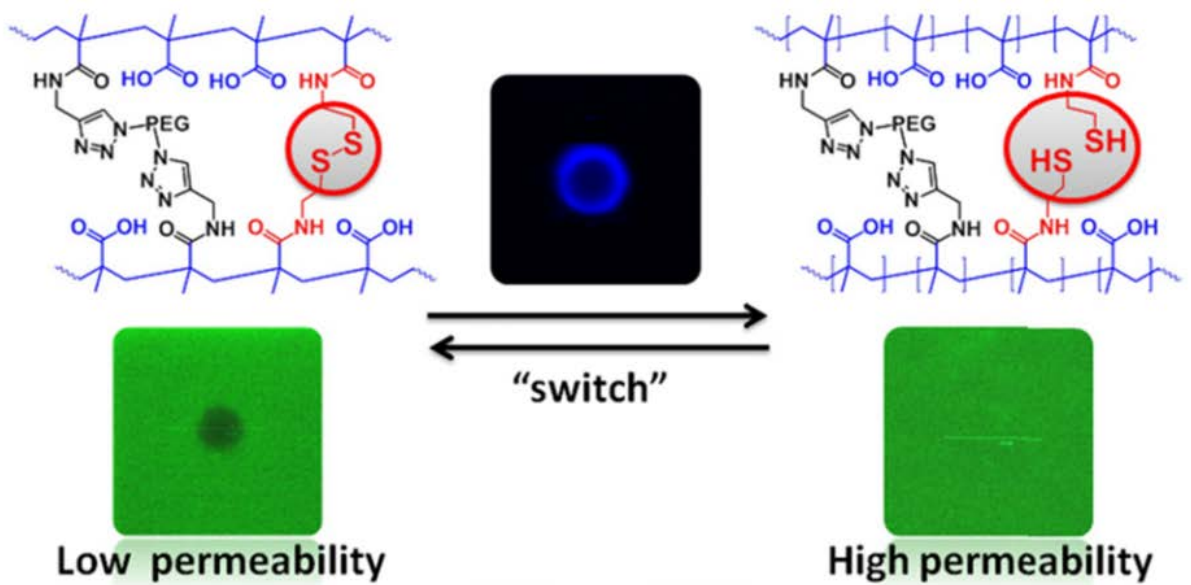

